\title{
When access is an issue: exploring barriers to predictive testing for Huntington disease in British Columbia, Canada
}

\begin{abstract}
Alice K Hawkins $^{\star 1}$, Susan Creighton ${ }^{1}$ and Michael R Hayden ${ }^{1}$
Predictive testing (PT) for Huntington disease (HD) requires several in-person appointments. This requirement may be a barrier to testing so that at risk individuals do not realize the potential benefits of PT. To understand the obstacles to PT in terms of the accessibility of services, as well as exploring mechanisms by which this issue may be addressed, we conducted an interview study of individuals at risk for HD throughout British Columbia, Canada. Results reveal that the accessibility of PT can be a barrier for two major reasons: distance and the inflexibility of the testing process. Distance is a structural barrier, and relates to the time and travel required to access PT, the financial and other opportunity costs associated with taking time away from work and family to attend appointments and the stress of navigating urban centers. The inflexibility of the testing process barrier relates to the emotional and psychological accessibility of PT. The results of the interview study reveal that there are access barriers to PT that deter individuals from receiving the support, information and counseling they require. What makes accessibility of PT services important is not just that it may result in differences in quality of life and care, but because these differences may be addressed with creative and adaptable solutions in the delivery of genetic services. The study findings underscore the need for us to rethink and personalize the way we deliver such services to improve access issues to prevent inequities in the health care system.
\end{abstract}

European Journal of Human Genetics (2013) 21, 148-153; doi:10.1038/ejhg.2012.147; published online 11 July 2012

Keywords: Huntington disease; predictive testing; telehealth

\section{INTRODUCTION}

Huntington disease (HD), is a neurodegenerative disorder characterized by chorea, dementia, personality and mood disturbances. ${ }^{1}$ It is inherited in an autosomal dominant manner and is caused by a CAG expansion mutation in the $H D$ gene. $^{2}$ Although age of onset varies, the mean age of first symptoms is $35-50$ years. There is a progression of the cognitive, movement and psychiatric symptoms with death usually occurring 15-20 years after first symptoms. ${ }^{1,3}$ There is no treatment to alter disease course, however, standards of care for managing HD do exist. ${ }^{4}$

Predictive testing (PT) for the disorder has been available since 1986 (via linkage) ${ }^{5}$ and via direct mutation testing since $1993 .{ }^{6} \mathrm{PT}$ to determine risk status enables people to access benefits including: relieving uncertainty, reproductive planning, improved ability to plan for the future, access to support and research opportunities. ${ }^{7-9}$ In keeping with the HD PT guidelines, ${ }^{10}$ the PT process in British Columbia (BC), Canada usually involves three to four in-person appointments at the Center for HD in Vancouver to undergo physical exam, genetic counseling and education, psychosocial evaluation, results and follow-up ${ }^{10,11}$ (Table 1 ). This process usually takes 4 weeks from initial appointment to the results session, and individuals are encouraged to bring a support person (eg, spouse), particularly to the results session (though this is not required). Depending on specific circumstances (including distance to Vancouver) this protocol may be adapted so that only one appointment occurs in Vancouver, with the remainder of the sessions and results being provided by a local GP with the guidance and support of the Center for HD genetic counselor. The protocol was designed to promote informed decision making and to help protect individuals from the potential for serious psychological damage of receiving HD test results.

Despite the availability of comprehensive PT programs, PT rates among individuals at risk for HD are lower than initially expected ${ }^{12,13}$ with reported uptake rates ranging from 5 to $25 \% .^{9,12,14,15}$ Although PT for HD should be a carefully considered individual choice, there may be barriers to testing so that at risk individuals who would like to pursue testing cannot realize the potential benefits of PT. ${ }^{16,17}$ Although the cost of PT is likely to be a significant barrier to testing in countries where PT is not covered by the healthcare system (PT is covered in Canada), other barriers to PT are likely to exist. One such barrier, particularly relevant in $\mathrm{BC}$, may be the accessibility of genetic services. ${ }^{18-20}$ The PT uptake rate in BC is $25 \%$. A review of PT records from the $\mathrm{BC}$ reveals that testing rates are lower in rural areas (areas $>2 \mathrm{~h}$ drive from the PT clinic) than non-rural areas with PT uptake rates of those at $50 \%$ risk for $\mathrm{HD}$ at $30 \%$ for non-rural populations and $16 \%$ for rural populations (unpublished data). These numbers are based on the actual number of tests in the study period/ actual number of individuals at $50 \%$ risk and eligible for testing to avoid the recognized difficulties in accurate uptake calculations. ${ }^{14}$ These data suggest that access may be a barrier to testing, particularly for those living in rural areas. 
Table 1 PT process in BC

\begin{tabular}{|c|c|c|}
\hline Appointment & Timeline & Topics covered/session content \\
\hline $\begin{array}{l}\text { 1: General coun- } \\
\text { seling session }\end{array}$ & Week 1 & $\begin{array}{l}\text { 1. Psychological Screening } \\
\text { Questionnaires } \\
\text { 2. Neurological exam (if possible) } \\
\text { 3. Genetic counseling and info about } \\
\text { PT and PT protocol } \\
\text { 4. Reasons for PT } \\
\text { 5. Exploration of family and social } \\
\text { support }\end{array}$ \\
\hline $\begin{array}{l}\text { 2: Pre-results } \\
\text { session }\end{array}$ & Week 3-4 & $\begin{array}{l}\text { 1. Participant's decision } \\
\text { 2. Psychological and social prepara- } \\
\text { tion for results }\end{array}$ \\
\hline 3: Results session & Week 4-5 & Results of PT \\
\hline $\begin{array}{l}\text { 4: Post-results } \\
\text { sessions }\end{array}$ & $\begin{array}{l}\text { Week } 6-7 \text { and } 6 \\
\text { months post results }\end{array}$ & $\begin{array}{l}\text { Integration of results, coping and } \\
\text { further support }\end{array}$ \\
\hline
\end{tabular}

Abbreviation: PT, predictive testing.

To understand and explore whether accessibility of PT services is a barrier to testing, we conducted an interview study of individuals at risk for $\mathrm{HD}$ throughout $\mathrm{BC}$. BC has a population of approximately 4.5 million people, 2.5 million of whom reside within a $2 \mathrm{~h}$ drive of downtown Vancouver. ${ }^{21}$ The remainder of the population is dispersed and may have to travel for up to $24 \mathrm{~h}$ to reach the PT center in Vancouver. This research sought to understand the obstacles to testing in terms of the accessibility of services in Vancouver, as well as exploring the mechanisms by which this issue may be addressed.

\section{MATERIALS AND METHODS}

\section{Study sample and recruitment}

Interview recruitment was based on a non-probability sample with respondents chosen based on availability and convenience. Recruitment aimed for a diverse sample stratified for location (rural versus non rural), testing status (tested versus not tested) and test result (CAG in the disease range versus normal CAG size). These categories aimed to ensure a range of viewpoints and to minimize bias toward a particular sub-group (eg, individuals with a highrisk result might perceive the process differently than someone with a normal result). Although every effort was made to obtain a similar number of participants in each stratification, this was not always possible because of: (1) the demographic distribution of $\mathrm{BC}$; (2) people who had not undergone PT were more difficult to recruit as they were not as likely to be known (eg, not on relevant mailing lists); and (3) of those tested, individuals who had a CAG expansion were more likely to be engaged and willing to be involved in research (those who received normal result were less likely to stay active in HD research).

Recruitment involved a multi-pronged approach. First, patients who had gone through PT within 5 years through the Center for HD were contacted via letter and returned a form if willing to participate. Second, to access those at risk but not tested, we sent a notice regarding the study via the Huntington Society of Canada, BC Chapter newsletter and also to those on the Center for HD study database. Finally, we used snowball sampling to recruit family members of those who had already been interviewed. After initial interest was confirmed, all participants were asked to sign a written consent form agreeing to participate. Research ethics approval was obtained for this study.

\section{Data collection}

Interviews were conducted in person or via phone and usually lasted 45-60 min. Interviews consisted of open-ended questions focussing on: (1) perceptions of the PT process; (2) experience of considering and/or undergoing PT; (3) barriers to testing; (4) assessment of the key components of the PT process; and (5) opinions regarding the provision of remote PT. Interviews were tailored based on whether the respondent had undergone $\mathrm{PT}$, their location and their at risk status (if known). Interviews were conducted until theoretical saturation was reached, which occurs when additional interviews provide repeated themes but no further insights. ${ }^{22,23}$

\section{Data analysis}

All interviews were recorded, transcribed and entered into analysis software (NVIVO 9, QSR International, Cambridge, MA, USA). Transcripts were read several times to generate categories of information and reveal prominent themes. Data were then coded for themes and subject to thematic analysis, adjusting coding methods until saturation was reached. ${ }^{22}$ The interview, transcription and coding process occurred concurrently so that emerging concepts or questions informed subsequent interviews to improve focus, clarity and granularity on specific issues. ${ }^{24,25}$ This analysis method, known as constant comparison, ${ }^{26}$ allows a conceptual framework to be developed, and subsequently refined and verified, to ensure the final categories form a comprehensive model of the data.

\section{RESULTS}

\section{Sample characteristics}

A total of 33 interviews were conducted for this study, with participants stratified by location, testing and result status, as outlined in Figure 1.

Accessibility of PT services as a barrier to testing. Results of the interview study reveal that the accessibility of PT is a significant barrier for those considering PT for HD in BC. Detailed analysis of the interview transcripts reveals that accessibility is a complex barrier, and can be broken down into two major sub-barriers or themes. The first of these, distance, is structural in nature and relates to difficulties such as travel, financial and opportunity costs of attending sessions, stress related to travel and distance from support. The second major sub-barrier that emerged relates to the accessibility of the actual testing process including counseling and support person requirements, the structure of the results session and the process length. These barriers and sub-barriers are outlined in Figure 2, and described in more detail below. Direct interview participant quotations have been used where relevant to highlight specific issues. Although this discussion focuses on access barriers to PT, factors that improved access (such as travel reimbursement and use of local GPs to deliver results) have also been highlighted.

\section{Distance}

The first major barrier in accessing PT that emerged through the interview analysis related to distance to the clinic in Vancouver.

Travel requirement and time involved. Perhaps the most obvious access barrier is the necessity of traveling to undergo the appointments typically involved in the PT process. This was of particular concern for those from rural communities who need to take a variety of transportation (eg, airplane, ferry and bus) for long periods. As one rural respondent (not-tested) explains, this can be a big commitment: 'We'd get up at 4 o'clock, we wouldn't get back till 10 o'clock that night....that was a lot, that was a big commitment.' Others noted the financial and time concerns involved with such travel: 'not everybody is going to be willing to travel like that. Some people can't afford it, some people just don't have the time'. Another participant reported delaying results for 2 years because of distance and travel time: 'I would have had to take so much time off work that it would have cost me a lot of money to go to Vancouver. The whole reason we ended up going in March was because we were going on holiday and we decided 


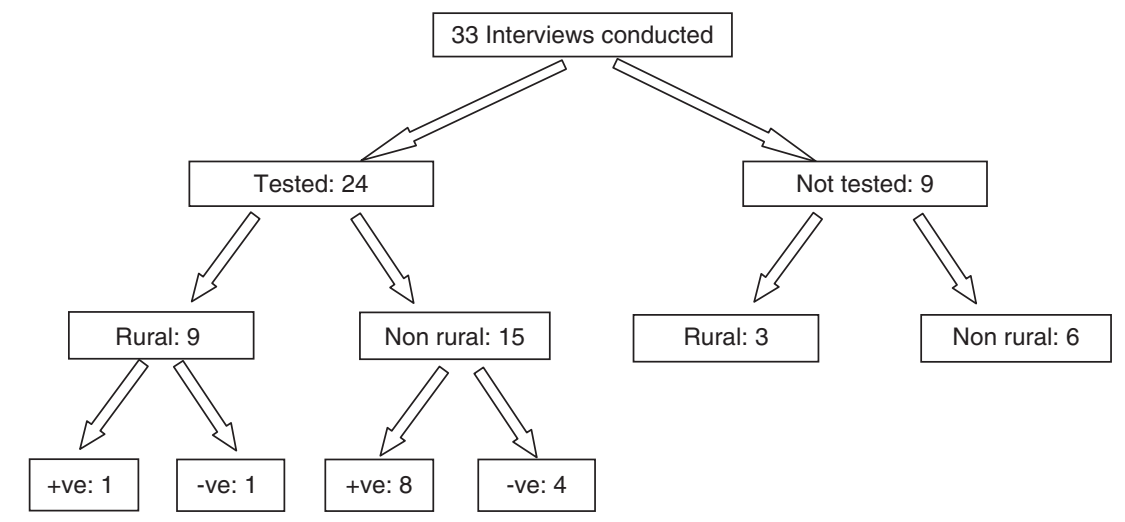

Figure 1 Interview participant characteristics.

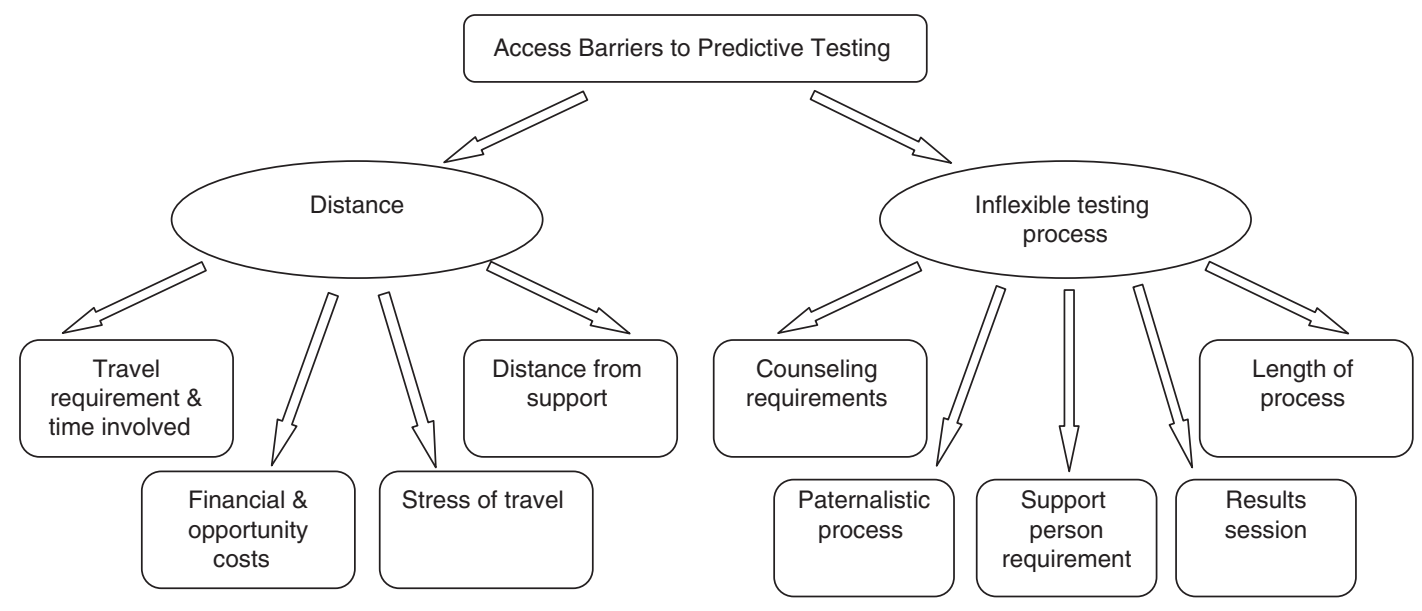

Figure 2 Access barriers to PT; themes revealed in the interview analysis.

to try to fly through Vancouver... so the process actually made me wait longer to go ahead with my results'.

Several interview respondents noted that siblings and other family members living in more rural communities had not undergone testing and that the 'main drawback for them was distance'. As one individual living close to Vancouver explained, 'my dad was...living in a rural area, very rural. He had to come (to the clinic) and it was just terrible. He had to keep coming back and forth and it was really hard on him... He left... being told that he had Huntington's...I made him stay with us for four days...it's an eight hour drive on a long road.'

Financial and opportunity costs. Although those in BC from rural areas may be eligible for provincial financial support and assistance in traveling to medical centers, this only goes some way to improving access. Shortcomings of this system were noted including length of reimbursement process and difficulties in obtaining and completing appropriate forms. Moreover, this assistance does not address lost opportunity costs including the need to take time away from work and family. This is particularly relevant for those with young children or caring for HD affected relatives. As one participant describes, these issues had a role in her decision not to undergo testing: 'I was a working mom. I couldn't take the time off to go down and do that... Distance definitely had a part (in not having testing).'

Stress of travel. Somewhat surprisingly, access was an issue even for those relatively close to Vancouver due to the stress of driving to the city and rush hour traffic that could result in a $3 \mathrm{~h}$ journey home. These individuals felt that they needed to take a whole day off for an appointment. As one respondent voiced 'it would make it easier if we didn't have to go to the clinic. (It) is forever... away.'

Distance from support. Others noted that when receiving results they had to travel to the HD clinic very early (or the day before) and then home at the end of the day. The individual receiving results usually had to attend the appointment alone (as the support person could not afford to take time off). They then faced a long journey home before they reached their support system to begin to deal with and process their results. One individual, who received results from his family doctor, described how important it had been for him to receive the results in his local community, on his terms (including bringing a beer to the results session): '(My doctor) said he'll make it the last appointment so there's no distractions and lots of time... we were there for a couple of hours and I drank my beer and he shook my hand and gave me a hug and apologized... But all the supports were in place. I knew exactly what the plan was.' This individual also noted that the possibility of having results delivered by his local GP (rather than the clinic in Vancouver) had facilitated his access to PT.

\section{Inflexible testing process}

The second major barrier that emerged from the interviews related to the accessibility of the actual testing process. These concerns related to the inflexible nature of the process, which did not take into account an individual's specific circumstances and needs. 
Length of the testing process. Interview respondents felt that the time it took to go through testing was lengthy and frustrating. The initial waiting period was a source of frustration for those who had 'made up their minds' and were 'on a mission' to receive results. As one interview respondent explained: 'Just tell me what I'm dealing with so I can move on and make plans'. Some respondents voiced this frustration more strongly: 'I hate waiting. I wanted to do it' and '(the waiting process is) just gut wrenching. Many participants, including tested and non-tested, also perceived the number of appointments to be somewhat unnecessary. This may have detrimental effects on other family members and relationships: 'I think the time frame was just too long. It doesn't need to be.... I just found it very frustrating to have to wait... And I came to a point - well my dad, he didn't even know. He died. I didn't even get to tell him actually - tell him that I tested positive. So it was really heartbreaking, because he died the same year.... I wanted him to know.'

Paternalistic process. Although the testing process was seen as too lengthy by some, several interview participants mentioned that in retrospect they understood why the process was so involved. Others felt the process could have been 'somehow optional or individually tailored' to the needs of the specific patient being seen. They felt that the process was somewhat paternalistic in that they already understood and knew the disease and the ramifications of PT. Some felt that the counseling before testing and psychological workup involved 'too much handholding' and that they were 'over babied.' Others felt there were too many questions regarding depression and suicide and 'a lot of things that I don't have... They dwelled on it a lot....I didn't think was necessary for me.'

Counseling requirements. Similarly, for both those who had and had not had testing, the length of the PT process, and counseling involved (whether real or perceived) was a barrier to testing. For example, one respondent recalled his reaction to hearing what was required: 'When we first got the protocol related to the testing and the three or four visits that were required and all of that, talking amongst my siblings, it was kind of like, why? Why do we have so many?' One individual who had not undergone testing explains this barrier further: 'I'm assuming that I'm going to be smothered with 'help' when I get my results back that are a positive that I do have HD'. The perception that the PT process was smothering had a role in this individuals' decision not to be tested.

Results session. The desire for a personalized approach is perhaps best illustrated by viewpoints on the results session. Respondents felt that the way results are delivered is a very personal preference. Most preferred receiving results in person, as they felt it was better to receive results from someone who they knew or had a personal connection to. Respondents voiced concern that phoned results, or delivery of results where you could not physically see the person, would make it difficult for the person delivering results to evaluate the well-being and emotional state of the person receiving results. As one respondent notes: 'for me the personal contact was crucial' that felt 'almost like a cushion'. However, while most respondents indicated that they felt in person result reporting was 'preferable' many also acknowledged that for some people this would not be possible, or desirable. Some respondents said that if they had received results via phone they 'would have been just as okay as I was with it being done in person'.

There were mixed views as to whether it was preferable to receive results from a local family physician or from the HD testing center. For example, of those who said they preferred to be at the HD clinic when receiving results they indicated that they could 'trust the results', and they were getting the 'best of the best care' and 'expertise'. For those who thought it preferable to receive their results from their local GP a number of reasons were articulated including: being in a supportive environment (ie, close to home); the support person could easily attend; and having a good 'rapport' or history with their GP (so they felt comfortable/ trusted their GP). As one rural participant stated regarding receiving her result from her GP, 'it was better for me to be here and just come home and deal with it on my own.' However, some respondents voiced concern about the knowledge and expertise of their local GP in terms of providing counseling and results for HD PT.

Some respondents would prefer to receive results alone so they could process and reflect. One respondent felt strongly about privacy when receiving results and compared it to receiving a school report: 'It's like making somebody open their report card when it's not a good one in front of somebody... (and saying) 'Oh, let's see how she's going to respond.'... Did you open your report card at school among all your friends? I sure as heck didn't... (I want to) go home, I want to close the door, I want to see it and then go, 'Okay, I understand why I've got it or got that mark or got the positive. And okay, we'll put on a bright face and carry on.'

Support person requirement. Finally, some participants voiced annoyance at the requirement that a support person be present for results (this is usually standard practice for patients undergoing PT). Some felt that by saying they would rather receive results alone they would be incorrectly characterized as being 'in denial' or 'at risk' when actually they were just an independent person who preferred to 'just deal with it.' As one individual who decided not to proceed with testing explains: 'I don't want my husband hugging me and telling me, 'Oh, everything will be fine,' until I am fine with it... I would need... a period of time to process it myself.'

\section{DISCUSSION}

The results of the interview findings reveal that the accessibility of PT can be a barrier to testing for two major reasons: distance and the rigidity of the current testing process. The first of these, distance, is not unique to HD, and has been seen in other areas of healthcare, including other genetic services. For example, in an examination of hereditary cancer services, D'Agincourt-Canning noted that distance posed a major barrier for rural communities: 'Not only does it create an added financial burden (travel, accommodation/meals and time away from work) but people must leave their family and social supports to get the medical services they need.27 The second barrier that emerged from the data is the inflexibility of the testing process whereby the testing protocol itself may discourage testing. ${ }^{19}$

Together the barriers revealed by the study may act as substantial impediments to individuals receiving the counseling, support and information they require. Addressing these barriers is important on several different levels. First, appropriate access to PT services allows people autonomy in their healthcare decision-making. ${ }^{17}$ Although we do not advocate all those at risk for HD undergo PT, individuals at risk for the devastating condition need to be given the appropriate opportunity to discuss PT and receive information on the pros and cons of receiving results. In addition, failing to understand an individual's circumstances may have detrimental effects on the patient, as illustrated by the respondent who relayed the devastation at not being able to discuss her results with her father before he passed away.

The second fundamental reason we need to consider the accessibility of PT relates to broader considerations of equity in healthcare. 
Although inequity in the provision of specialist services is not a new phenomenon, nor unique to genetic services, consideration of solutions that mitigate these inequities is warranted. The current testing model may be restrictive and inflexible in its requirements and discriminates against those who do not access PT services for either physical/ structural reasons, or for emotional/ psychological reasons. Most obviously, this may prevent comprehensive PT. However, perception of PT (whether realistic or not) is also a concern as it influences participation in PT initiation or continuation.

Finally, these findings are relevant to other jurisdictions. Although BC's geography and the healthcare system differ from other areas of the world, the results are important specifically because they reveal accessibility of testing can be a barrier for diverse reasons. Barriers are not just relevant to those living in remote locations, as even those living within close proximity may encounter georgraphic, financial or other access deterrents. These results underscore the importance of understanding and exploring these factors on both a local and individual level. For example, despite the fact that BC has a relatively dispersed populous, it has one of the highest uptake rates of PT, likely due to factors such as universal healthcare coverage and the prominence and history of research and clinical care provided by the Center for HD. These contextual factors are also important in understanding differences in uptake rates of PT in other countries and jurisdictions, and only by examining potential barriers and contextual factors on a local level can we begin to address and remove these barriers.

Solutions to improve access to PT need to be flexible and take into account individuals' circumstances and needs. For example, the concern that the process is over-burdensome and smothering can be addressed via (1) education regarding PT and (2) adapting and personalizing the testing process to an individual's circumstances. ${ }^{16}$ In addition, the finding that some participants felt receiving results from their local GP had been (or would be) successful, illustrates another potential mechanism to improve access. Creating solutions also requires recognition that access barriers are complex issues that may not be resolved by, for example, simply providing travel assistance (although this did help improve access to some extent). Moreover the role of other established reasons why people do not pursue PT should not be negated. These factors, including views about controlling the future, family attitudes and norms, financial/insurance implications, the impact on others and the potential for discrimination are recognized in having an important role in the decision-making process. ${ }^{28,29}$ As such, the aim of mechanisms to reduce access barriers is to ensure that such barriers are not the only reason that individuals do not pursue testing.

PT can be provided relatively inexpensively and effectively via novel telecommunication methods such as videoconferencing. Telemedicine in genetics is not new, and has been successfully used to improve access in other areas that require complex counseling and decision making, such as hereditary cancer. ${ }^{30-33}$ Moreover studies suggest that telegenetics is successful and preferred by some individuals, and may also result in more cost-effective services. Telemedicine also has potential to improve access to services for those in less developed nations, ${ }^{34}$ where access to specialized genetic professionals may be limited, at best. As a result of this interview study, BC is now evaluating the provision of PT via telehealth, with the support of a local GP. This telehealth model is in keeping with the new PT guidelines for HD (Macleod et al, in press), and preliminary analysis reveals patient satisfaction and well-being measures throughout the PT process maintain the quality of care and support necessary for those undergoing PT.
This study has a number of limitations. First, we acknowledge that results may not be applicable in other healthcare regions. However, given the low number of genetics professionals, and concentration of such professionals in large, urban, academic medical centres ${ }^{35}$ it is likely that these access issues are similar in other jurisdictions. Second, owing to the nature of the study, recruitment could not ensure a random sample of those at risk for PT was selected, and it was particularly difficult to find participants in rural areas who had not been tested. This constraint may also indicate the difficulty in accessing such individuals, not just to participate in research studies, but also in clinical care. Further studies may help elucidate the true meaning of access barriers in these harder to reach communities. In addition, recruitment was inherently biased toward those who self-selected to discuss these issues, and it is difficult to access individuals who have not had testing (as they are not always known) or those who had received results within the normal CAG range (as these individuals may have put HD behind them). However, snowball sampling methods (ie, asking individuals to inform family members who were not tested or those who had received normal results about the study) and conducting interviews until saturation appeared to be met helped us to recruit a sample representing a diversity of views.

This research sought to understand the obstacles to testing in terms of access to genetic services, as well as exploring the mechanisms by which this issue may be addressed. Such research is essential to decrease inequalities in access to PT services, and other potentially beneficial research advancements in the field of $\mathrm{HD}$ such as involvement in clinical trials and knowledge of new therapies. What makes accessibility of PT services for HD important is not just that it may result in differences in quality of life and care, but because these differences may be addressed with creative and adaptable solutions in the delivery of PT services. The study findings underscore the need for us to rethink and personalize the way we deliver such services to improve access issues to prevent inequities in the health care system. This issue is pertinent for other areas of genetic medicine to ensure that genetic technologies and testing benefit all. ${ }^{36}$ However, access barriers remain largely unaddressed, despite the increasing importance of personalized medicine and technological developments that decrease the cost and improve portability of genetic tests and other healthcare technologies. Telemedicine, supporting GPs and other healthcare providers to assist in the provision of PT and tailoring testing protocols are just some of the ways by which we may ensure the benefits of PT are more widely realized.

\section{CONFLICT OF INTEREST}

The authors declare no conflict of interest.

\section{ACKNOWLEDGEMENTS}

We thank the study participants who shared their thoughts and experiences with us. The authors receive support for research into PT for HD from the CHDI and CIHR.

\footnotetext{
1 Walker FO: Huntington's disease. Lancet 2007; 369: 218-228.

2 MacDonald ME, Ambrose CM, Duyao MP et al: A novel gene containing a trinucleotide repeat that is expanded and unstable on Huntington's disease chromosomes. Cell 1993; 72: 971-983.

3 Hayden M: Huntington's Chorea. New York, NY, USA: Springer-Verlag, 1981

4 Rae D, Simpson SA: A standard of care for Huntington's disease: who, what and why. Neurodegen Dis Manage 2012; 2: 1-5.
} 
5 Brandt J, Quaid KA, Folstein SE et al: Presymptomatic diagnosis of delayed-onset disease with linked DNA markers. The experience in Huntington's disease. JAMA 1989; 261: 3108.

6 Kremer B, Goldberg P, Andrew SE et al: A worldwide study of the Huntington's disease mutation: the sensitivity and specificity of measuring CAG repeats. New Engl J Med 1994; 330: 1401-1406.

7 Wiggins S, Whyte $\mathrm{P}$, Huggins $\mathrm{M}$ et al: The psychological consequences of predictive testing for Huntington's disease. NEJM 1992; 327: 1401-1405.

8 Codori AM, Brandt J: Psychological costs and benefits of predictive testing for Huntington's disease. Am J Med Genet 1994; 54: 174-174.

9 Creighton S, Almqvist EW, MacGregor D et al: Predictive, pre-natal and diagnostic genetic testing for Huntington's disease: the experience in Canada from 1987 to 2000. Clin Genet 2003; 63: 462-475.

10 Went L: Guidelines for the molecular genetics predictive test in Huntington's disease. J Med Genet 1994; 31: 555-559.

11 Benjamin CM, Adam S, Wiggins S et al: Proceed with care: direct predictive testing for Huntington disease. Am J Hum Genet 1994; 55: 606.

12 Hayden M: Predictive testing for Huntington's disease: the calm after the storm. Lancet 2000; 356: 1944-1945.

13 Meissen GJ, Berchek RL: Intended use of predictive testing by those at risk for Huntington disease. Am J Med Genet 1987; 26: 283-293.

14 Tassicker RJ, Teltscher B, Trembath MK et al: Problems assessing uptake of Huntington disease predictive testing and a proposed solution. Eur J Hum Genet 2008; 17: 66-70.

15 Morrison P, Harding-Lester S, Bradley A: Uptake of Huntington disease predictive testing in a complete population. Clinical Genetics 2011; 80: 281-286.

16 Hawkins AK, Hayden MR: A grand challenge: providing benefits of clinical genetics to those in need. Genet Med 2011; 13: 197.

17 Hawkins AK, Ho A, Hayden MR: Lessons from predictive testing for Huntington disease: 25 years on. J Med Genet 2011; 48: 649-650.

18 Evers-Kiebooms G, Decruyenaere M: Predictive testing for Huntington's disease: a challenge for persons at risk and for professionals. Patient Educ Couns 1998; 35: 15-26.

19 Kessler S: Predictive testing for Huntington disease: a psychologist's view. Am J Med Genet 1994; 54: 161-166.

20 Demyttenaere K, Evers-Kiebooms G, Decruyenaere M: Pitfalls in counseling for predictive testing in Huntington disease. Birth Defects Orig Art Ser 1992; 28: 105.
21 Statistics Canada. 2010 Census Data retrieved 10 March 2012 from http://www. statcan.gc.ca/start-debut-eng.html, (Ottawa, ON, Canada).

22 Creswell J: Research Design: Qualitative, Quantitative, and Mixed Method Approaches, 2nd edn. Thousand Oaks, CA, USA: Sage Publications, 2003.

23 Strauss A, Corbin J: Basics of Qualitative Research, 2nd edn. Thousand Oaks, CA, USA: Sage Publications, 1998.

24 Charmaz K: Constructing Grounded Theory: A Practical Guide Through Qualitative Analysis. Thousand Oaks, CA, USA: Sage Publications, 2006.

25 Starks H, Brown Trinidad S: Choose your method: a comparison of phenomenology, discourse analysis, and grounded theory. Qual Health Res 2007; 17: 1372.

26 Corbin J, Strauss A: Basics of Qualitative Research: Techniques and Procedures for Developing Grounded Theory. Thousand Oaks, CA, USA: Sage Publications, 2008.

27 d'Agincourt-Canning L: Genetic testing for hereditary cancer: Challenges to ethical care in rural and remote communities. HEC Forum 2004; 16: 222-233.

28 Bombard Y, Veenstra G, Friedman J et al: Perceptions of genetic discrimination among people at risk for Huntington's disease: a cross sectional survey. Br Med J 2009; 338: b2175.

29 Binedell J, Soldan JR, Harper PS: Predictive testing for Huntington's disease: II. Qualitative findings from a study of uptake in South Wales. Clin Genet, 1998; 54: 489-496.

30 Gattas M, MacMillan J, Meinecke I, Loane M, Wootton R: Telemedicine and clinical genetics: establishing a successful service. J Telemed Telecare 2001; 7: 68 .

31 Coelho J, Arnold A, Nayler J, Tischkowitz M, MacKay J: An assessment of the efficacy of cancer genetic counselling using real-time videoconferencing technology (telemedicine) compared to face-to-face consultations. Eur J Cancer 2005; 41: 2257-2261.

32 Baumanis L, Evans J, Callanan N, Susswein L: Telephoned BRCA1/2 genetic test results: prevalence, practice, and patient satisfaction. J Genet Couns 2009; 18: 447-463.

33 Stalker $\mathrm{H}$, Wilson $\mathrm{R}$, McCune $\mathrm{H}$ et al: Telegenetic medicine: improved access to services in an underserved area. J Telemed Telecare 2006; 12: 182.

34 Wootton R, Bonnardot L: In what circumstances is telemedicine appropriate in the developing world? JRSM Short Reports 2010, p1.

35 National Human Genome Research Institute: The Future of Genomic Medicine: Policy Implications for Research and Medicine, 2006; http://www.genome.gov/17516574.

36 Collins FS, Green ED, Guttmacher AE, Guyer MS\& the National Human Genome Research Institute. A vision for the future of genomics research. Nature 2003; 422: 835-847. 